\title{
A New Alternative for Obtaining Nanocrystalline Bioactive Coatings: Study of Hydroxyapatite Deposition Mechanisms by Cold Gas Spraying
}

\author{
Núria Cinca ${ }^{\ddagger}, \dagger$ Anna M. Vilardell, ${ }^{\ddagger}$ Sergi Dosta, ${ }^{\ddagger}$ Amadeu Concustell, ${ }^{\ddagger}$ Irene Garcia Cano, \\ Josep Maria Guilemany, ${ }^{\ddagger}$ Sonia Estradé, ${ }^{\S}$ Alicia Ruiz, ${ }^{\S}$ and Francesca Peiró ${ }^{\S}$ \\ *Thermal Spray Center (CPT). Dpt. Ciència dels Materials i Enginyeria Metal.lúrgica, Universitat de Barcelona, c/Martí i \\ Franquès 1, E-08028 Barcelona, Spain
}

${ }^{\S}$ LENS-MIND/IN2UB, Dept. d'Electrònica, Universitat de Barcelona, c/Martí i Franquès 1, 08028 Barcelona, Spain

\begin{abstract}
The present article is intended to study the deposition mechanisms of bioactive hydroxyapatite (HA) particles by means of Cold Gas Spraying (CGS). A comparison of the deposition on two different substrates (Ti6Al4V and Al7075T6) and different particle sizes is presented. Although this is a more specific deposition technique for ductile materials, it is here shown that, in certain conditions, ceramic deposition is possible despite the inherent low ductility. The resulting internal structure and the features at the particle-substrate interface are discussed in view of Transmission Electron Microscopy examinations of a Focused Ion Beam lift-out prepared sample. Mainly, under shock compressive loading, the porous sintered powder proceeds through pore collapse, fragmentation and densification as well as grain refinement. The process is described through different plastic mechanisms in ceramics. This opens a new alternative route to produce nanocrystalline $\mathrm{HA}$ coatings through a cost-effective process.
\end{abstract}

\section{Introduction}

$\mathbf{P}$ LASMA Spraying (PS) of hydroxyapatite (HA) coatings is one of the most common ways to achieve bioactive surfaces for orthopedic implant prosthesis. In front of other existing technologies, it offers high deposition rates at low cost. ${ }^{1}$ Because of this, it is the most extended route from the commercial point of view; most hip stems, acetabular components, etc., are covered by these kind of coatings that promote rapid fixation and stronger bonding between the implant and the bone limiting the formation of any fibrous film.

One of the handicaps, however, of PS is the thermal decomposition of the feedstock material, resulting in the occurrence of many phases with different solubility ranges. ${ }^{1}$ This is beneficial considering the ion release to the surrounding medium because it promotes osseointegration; one noticeable problem is, however, produced when fast-dissolution phases are formed at the substrate (i.e., titanium) - HA coating interface, leading to possible coating detachment before complete bone resorption. Therefore, more recently, some research groups have seen in the Cold Gas Spray (CGS) technique a promising process to produce HA coatings without alteration of the initial composition of the spraying material. ${ }^{2,3}$ A successful coating deposited via CGS from

A. Bandyopadhyay — contributing editor

Manuscript No. 36855. Received May 7, 2015; approved November 6, 2015.

Manuscript No. 36855. Received May 7, 2015; approved November 6,

Corrections added on 19 February 2016, after first online publication.
†uthor to whom correspondence should be addressed. e-mail: ncinca@ $@$ cptub.eu controlled powder crystallinity, would allow producing bioactive coatings with controlled mineralization efficiency.

CGS is a solid-state process, where the feedstock powders are deposited at supersonic velocities $(200-1100 \mathrm{~m} / \mathrm{s})$ with minimum thermal effects on its nominal composition. Compared to the conventional thermal spray technologies, the material is not melted and mechanically anchored after shrinkage during rapid solidification, but rather more common metal sprayed materials are plastically deformed leading to dense and oxidation-free deposits. ${ }^{4-6}$ Ceramic particles have been commonly deposited either as cermets, ${ }^{7,8}$ or by codeposition with other ductile materials, i.e., HA has been more commonly codeposited with titanium. ${ }^{9-11}$

Due to the inherent brittleness of ceramics, their direct deposition has led to many less attempts ${ }^{12-16}$ and neither of those authors has been completely successful on clarifying the bonding mechanisms. Some of them have suggested that the attachment of the ceramic particles takes places mainly by the plastic deformation of the metallic substrate with particle fracturing, thus remaining ceramic portions embed within the ductile surface; ${ }^{12-15}$ by contrast, Yamada et al. ${ }^{16}$ reported thick $\mathrm{TiO}_{2}$ coatings onto metallic and ceramics substrates and their results support a chemical bonding rather than a mechanical anchoring. The inherent feedstock structure of the powder used by these authors seems to be crucial for the success of their work; this consisted in a porous microsized powder with nanoscaled agglomerated particles.

Two low-temperature powder-based dry manufacturing processes have succeeded in ceramic deposition although their research is within the initial steps; they are aerosol deposition (AD) and nanoparticle deposition system (NPDS), which appeared in the 1990 s and 2000s, respectively. AD involves aerosol formation of submicrometer particles, while in NPDS, nanosized or microsized powder is sprayed at room temperature under low-vacuum conditions to achieve microscale deposition. Fragmentation and compaction of submicrometer particles into nanoparticles by impact has been reported to be the main bonding mechanism in such processes. $^{17-19}$

Different numerical and simulation studies have been tested to optimize conditions for HA deposition in CGS. Zhang et al. ${ }^{20}$ studied the factors influencing HA particle acceleration using a computational fluid dynamics program FLUENT. Singh et al. used the Taguchi method to optimize HA conditions in $\mathrm{CGS}^{21}$ and found that gas type and particle diameter were the most influencing variables.

The successful build-up of a dense metal coating produced by CGS is well-known to be dependent on the metallurgical bonding between particles, which is influenced by the local conditions at the corresponding interfaces i.e., particle-particle and particle-substrate. The high kinetic energy of the particles is transformed into heat and plastic deformation, leading to plastic flow and the well-known "adiabatic shear 
instabilities", when thermal softening is predominant over work hardening of the material. ${ }^{22}$ In order to evaluate the influence of the temperature and strain rate on the microstructure of a hot-worked material, some processing maps have been studied for many different materials. ${ }^{23}$ Gandhi et al. ${ }^{24}$ overviewed the processing maps of several ceramics, therefore highlighting the different fracture mechanisms. The nature itself of the feedstock powder morphology involves another level of complexity on the understanding of such mechanisms.

Shock loading of granular materials effectively changes the state of the material and produces an effect similar to a phase change; i.e., during loading, the material does not follow a straight (or Rayleigh) line from its initial state $\left(p_{0}, V_{0}\right)$ to its $\left(p_{\mathrm{f}}, V_{\mathrm{f}}\right)$ final state (described here by pressure $p$ and volume $V$ ). Based on this, a failure criterion has to be included for the simulation of shock compaction processes. ${ }^{17}$ Failure mechanisms are actually closely associated to whether the impact velocity is such that the induced stress is below the Hugoniot Elastic limit (HEL). ${ }^{25}$ In addition, for granular/powder materials, the shock response also strongly depends on the shape and size of the particles. ${ }^{26}$

The present article investigates the deposition mechanisms of HA particles onto the typical bioinert Ti6Al4V alloy used for implants and a much softer alloy substrate i.e., A17075T6, for comparison of the deposition characteristics. The novelty of this works consists then not only on elucidating the structural changes of ceramics at the nanoscale level after shock loading during CGS, which is not clear from the literature, but also on introducing this concept to the interest of the biomedical field.

\section{Materials and Methods}

Single particles were sprayed onto mirror-like polished Ti6Al4V and 7075-T6 aluminum alloy substrates. In order to evaluate splat morphologies, wipe tests consisting of a fast movement of the substrate through the spray jet, were carried out.

A sintered powder from Plasma-Biotal Ltd. (Captal30, Derbyshire, UK) was used as feedstock. The Cold Gas Spray equipment was a KINETICS ${ }^{\circledR} 4000$ (Cold Gas Technology, Ampfing, Germany), with a maximum operating pressure of $40 \mathrm{bar}$, temperature of $800^{\circ} \mathrm{C}$, and limited to the use of nitrogen as the propellant gas. For this study, we used gas temperatures in the range of $200^{\circ} \mathrm{C}-600^{\circ} \mathrm{C}$, gas pressures 10 30 bar, and spraying distances from 10 to $60 \mathrm{~mm}$. More specific spraying conditions are not given in the present work because they are protected within an intellectual property). ${ }^{27}$

An initial examination of the mounted and polished cross sections of the splats was performed in a Scanning Electron Microscope (JEOL JSM 5310 microscope, Peabody, MA) operated at $20 \mathrm{kV}$ and equipped with an Energy-Dispersive Spectroscopy (EDS) for microanalysis. In order to examine the features within the particle and at particle-substrate interface, a thin lamella from a single splat was obtained by Focused Ion Beam (FIB) lift-out technique and compared to the initial feedstock structure. A FEI Strata Dual Beam 235 system was used. Much care was taken when irradiating the samples since some damage could be noticed through the preparation. Finally, transmission electron microscopy examinations were carried out using a $200 \mathrm{kV}$ JEM JEOL 2100 Transmission Electron Microscope. In addition, XRD measurements were done on a Bragg-Brentano $\theta / 2 \theta$ Siemens D500 diffractometer with $\mathrm{Cu} K_{\alpha}$ radiation; for the evaluation of oxidation, temperature-dependent X-ray scans were performed.

In order to evaluate the compression loading mode of the porous HA powder at different strain rates and the collapse effect of the porous structure, compression tests were carried out using a PicoIndenter PI85 nanoindenter (Hysitron Inc., Minneapolis) with a diamond flat punch of $10 \mu \mathrm{m}$ diameter under load control. The force-displacement curves were measured while recording the evolution in a Scanning electron Microscope. The nominal strain rate in the elastic regime was in the order of 10 and $100 \mathrm{~nm} \mathrm{~s}^{-1}$ considered as low and high-strain rates, respectively.

\section{Results}

\section{(1) Scanning and Transmission Electron Microscopy of the Feedstock Powder}

The micrographs in Fig. 1(a) display the morphology of the sintered HA powder; the magnified structure in Fig. 1(a) better shows the grain and porous morphology. This powder consists of spherical particles with particle size distribution of $-53+16 \mu \mathrm{m}$; these sintered particles are formed by small crystalline grains. The interconnected porous structure could be well observed through FIB along preparation time. No pore size extension was observed as result of the process preparation. Figure 1(b) shows the X-ray diffraction of the powder with very narrow peaks indicating the high crystallinity of the sample. It has been well identified according to the 9-432 pattern corresponding to a hexagonal $\mathrm{P} 63 / \mathrm{m}$ lattice.

The grain structure consisting in small equiaxed grains with an average grain size diameter of $0.5-2 \mu \mathrm{m}$ is illustrated in the TEM micrograph in Fig. 2; no exaggerated or anisotropic grain growth was observed in this powder. Some dense parts were seen with triple boundary points. Also in Fig. 2, the SAED pattern of a single grain is presented, showing a monocrystalline structure. Grain boundaries are well delineated by curved original flaws with a wavy nature. No amorphous secondary phase was observed at any of the triple
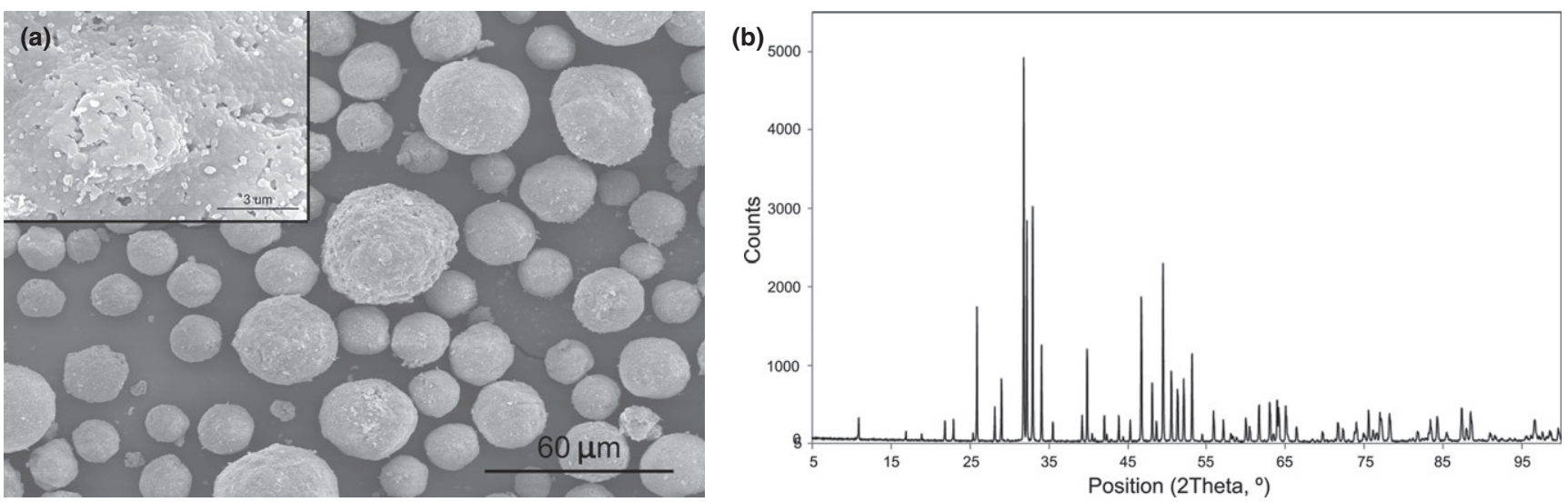

Fig. 1. (a) Morphology and (b) X-ray diffraction of the hydroxyapatite powder. 
points investigated, but rather Moiré fringes in addition to lattice fringes, resulting from the overlap of crystalline regions. Some grains are connected by necks, as also indicated by a black arrow. One of these neck-grain interfaces is also illustrated in the magnification of Fig. 2; it shows a bubble-like structure, which might come from some damage resulting from irradiation.

\section{(2) Stress-Strain Curves of Single Particles}

Figure 3 presents the compressive loading curves for an about two $15 \mu \mathrm{m}$ HA particles at low and high-strain rates. Although the high-strain rate is very much lower than that typically occurring during CGS $\left(10^{6} \mathrm{~s}^{-1}\right)$, it has been proved to be enough to show the collapse of the particle [Fig. 3(a)], whereas at low strain rate, the progressive cracking is observed by the several load drops during the indentation; the first significant drop corresponds to the occurrence of the crack indicated by an arrow in the inset of Fig. 3(b). At the end of the curve, just before the collapse, it can be observed how this upper right part becomes to be detached. By contrast, the insets in Fig. 3(a) indicate that the particles maintains intact until it cannot withstand the high load and collapses.

\section{(3) Single Impact Studies}

(A) Morphological Characterization: The different bonding mechanisms for soft and hard substrates were investigated by comparing single particle impact morphologies in so called "wipe tests". Figure 4 shows the splat morphologies

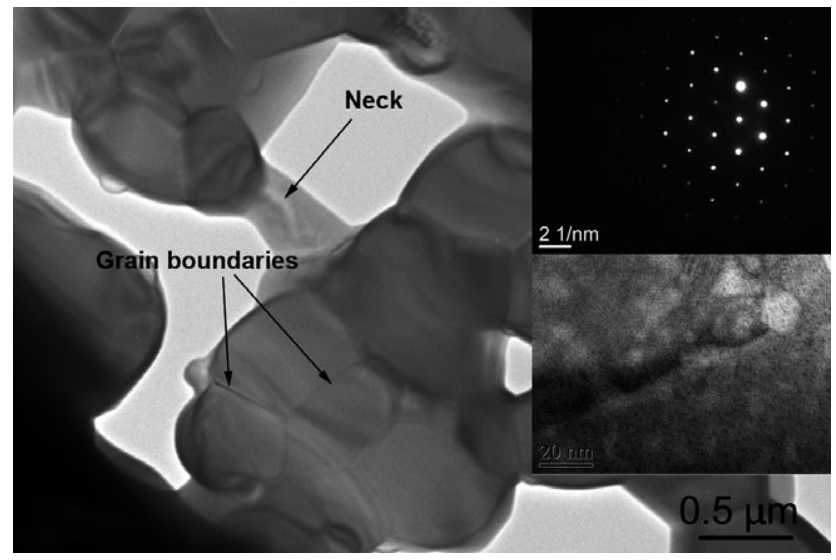

Fig. 2. TEM micrograph of the HA particle and the corresponding SAED pattern, also with detailed features of the neck-grain interface structure. of the Captal-HA powder onto the polished titanium alloy. It consists of a mixture of attached splats with fragments scattered around as well as ring-like shape HA remnants resulting from the bounce off of some of the particles. Near the fractured particles, slight substrate deformation was observed.

A very similar pattern is obtained when spraying onto the aluminum alloy, but this time, the substrate deformation is clearly visible (inset in Fig. 4) with jet morphologies resulting from its plastic flow. At higher magnification, even the imprint left by individual grains of the feedstock powder was revealed.

(B) Transmission Electron Microscopy of Cold Spray Hydroxyapatite Splats on Ti6Al4V: Figure 5 shows the FIB lamella of an approximately $12 \mu \mathrm{m}$ captal-HA splat onto the Ti6Al4V alloy. Figure 6 a shows the TEM micrograph of the corresponding central part, where the porous structure is still visible; the porous size distribution has changed because of the impact from that of the original particle since no more necks can be distinguished. This indicates that these narrow necks areas, if present, can act as damage initiation points under high-impact loading stresses. The appearance of contrast areas compared to Fig. 2 may be attributed to strained effects and also dislocation entanglement at grain boundaries. However, the grains still keep dimensions of the same order as those in feedstock. The structure keeps some pre-existing curved flaws and no extense intergranular cracking is noticed.

No large cracks were observed at all. Figure 6(b) exhibits a singular grain oriented along the [001] zone axis with a particular straight internal feature; its observation by HRTEM as well as its electron diffraction indicates that the whole grain is obtained in the same zone axis without any sign of misorientation. Therefore, there is no shear or twisting, just a plastic deformation feature in a single favorable oriented plane. Highly strained grains and fine grain areas are also characteristic of the complex structure resulting from impact [Fig. 6(c)]. More likely, selected area electron diffraction (SAED) indicates that most grains have a single-crystal structure, except for specific regions such as that indicated in Fig. 6(c), which appears to be highly polycrystalline and consists of randomly oriented crystallites less than $50 \mathrm{~nm}$ in size, with an average of $\sim 20 \mathrm{~nm}$.

The edge part of the splat appears to be more compact than the central part [Fig. 6(d)] and grains seem to be more cracked and slightly crushed; in some of them, extinction contours can be observed.

The particle-substrate interface is rather smooth with no noticeable detachment (Fig. 7). In addition, SAED pattern of the HA side shows a ring pattern typical of a polycrystalline area as well as the titanium side. From the HRTEM
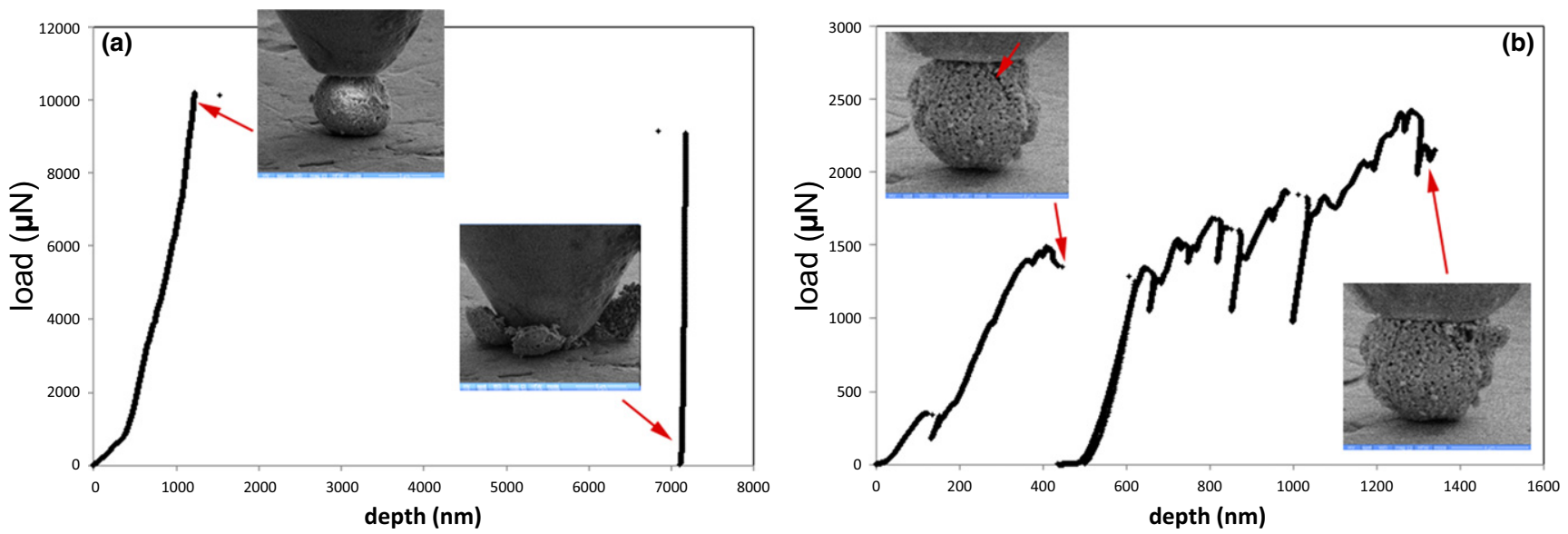

Fig. 3. Loading compressive curves for a single hydroxyapatite particle at (a) high and (b) low strain rate. 
image of the interface, many Moiré patterns can be observed, indicating overlapping of the fine grains. From the inserted FFT images (A-D), the one corresponding to the substrate side (D) presented some spots that were identified as the $\mathrm{TiO}_{2}$ phase. This would imply that the impact of the particle has not promoted the disruption of the titanium oxide layer.

Figure 8 is the general structure of a $7 \mu \mathrm{m}$ splat showing some single-crystal regions (label 1 with corresponding SAED at inset 1) and some polycrystalline areas (labels 2 and 3 with corresponding SAED at inset 2 and 3). The presence of both single crystal and fine-grain structures in the two investigated particles might be indicative of heterogeneous stress distribution upon particle impact.

Then, the impact mechanism of the $12 \mu \mathrm{m}$ HA splat seems to have occurred by: (i) slight void collapse, (ii) dynamic

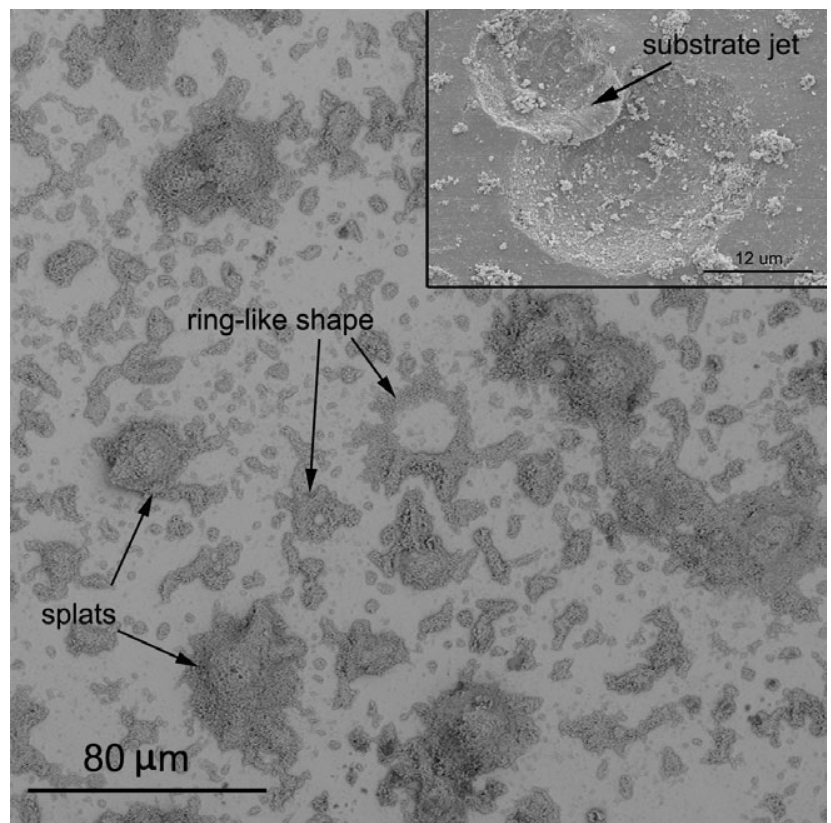

Fig. 4. Top view morphology of several HA single particle impacts on Ti6Al4V. Inset of the morphology of several craters left by single particle impacts on 7075-T6 Al alloy.

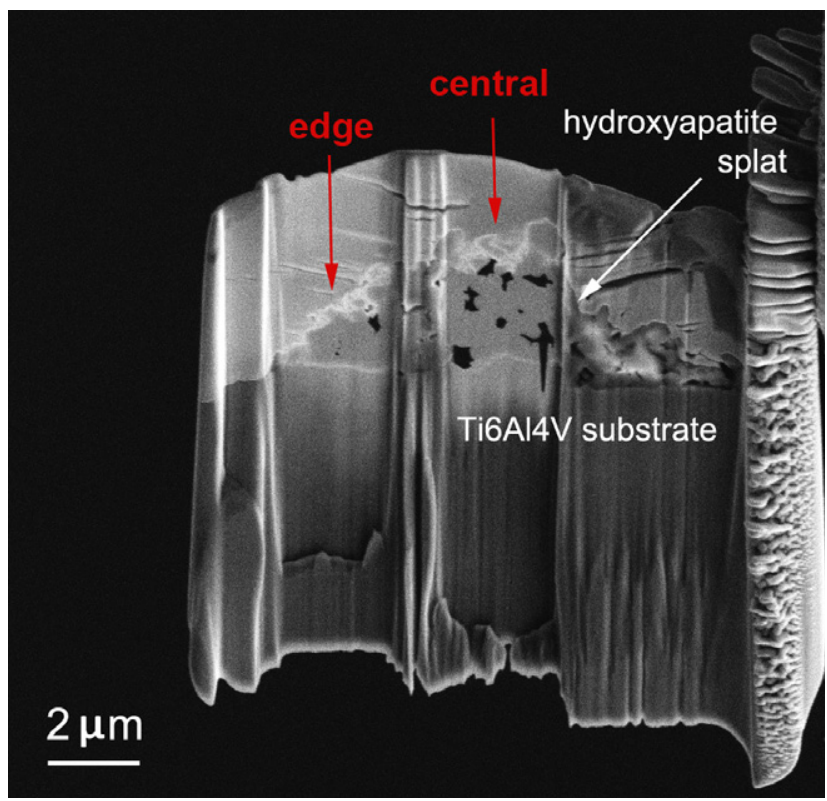

Fig. 5. FIB preparation of a captal HA splat on Ti6Al4V.
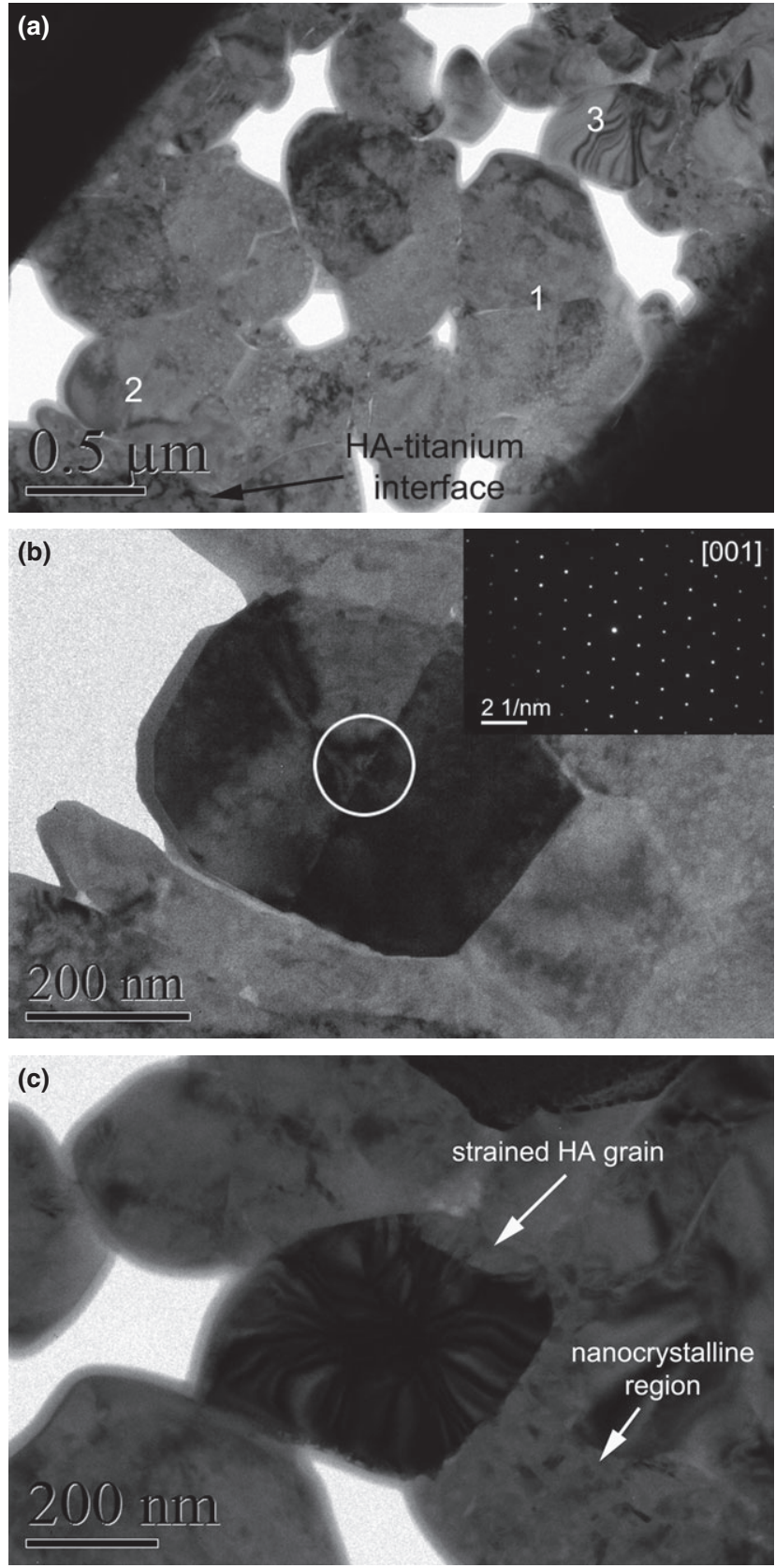

(d)

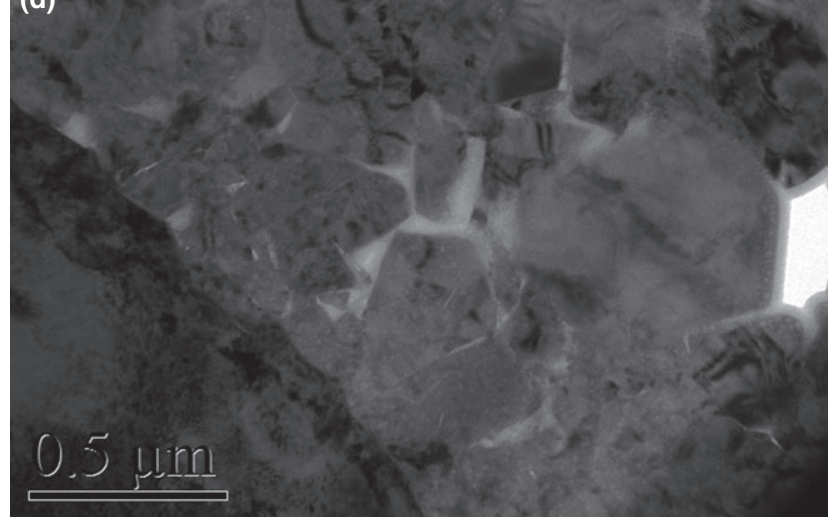

Fig. 6. TEM micrograph of the (a) central part of the HA splat onto Ti6Al4V, (b, c) detailed features corresponding to labels 1, 2, and 3 in "a", (d) more crushed areas at the edge of the splat.

fragmentation proceeding by cracking and crushing and (iii) reduction in crystal size by plastic deformation mechanisms. In view of the microstructure of the $7 \mu \mathrm{m}$ splat, a denser 

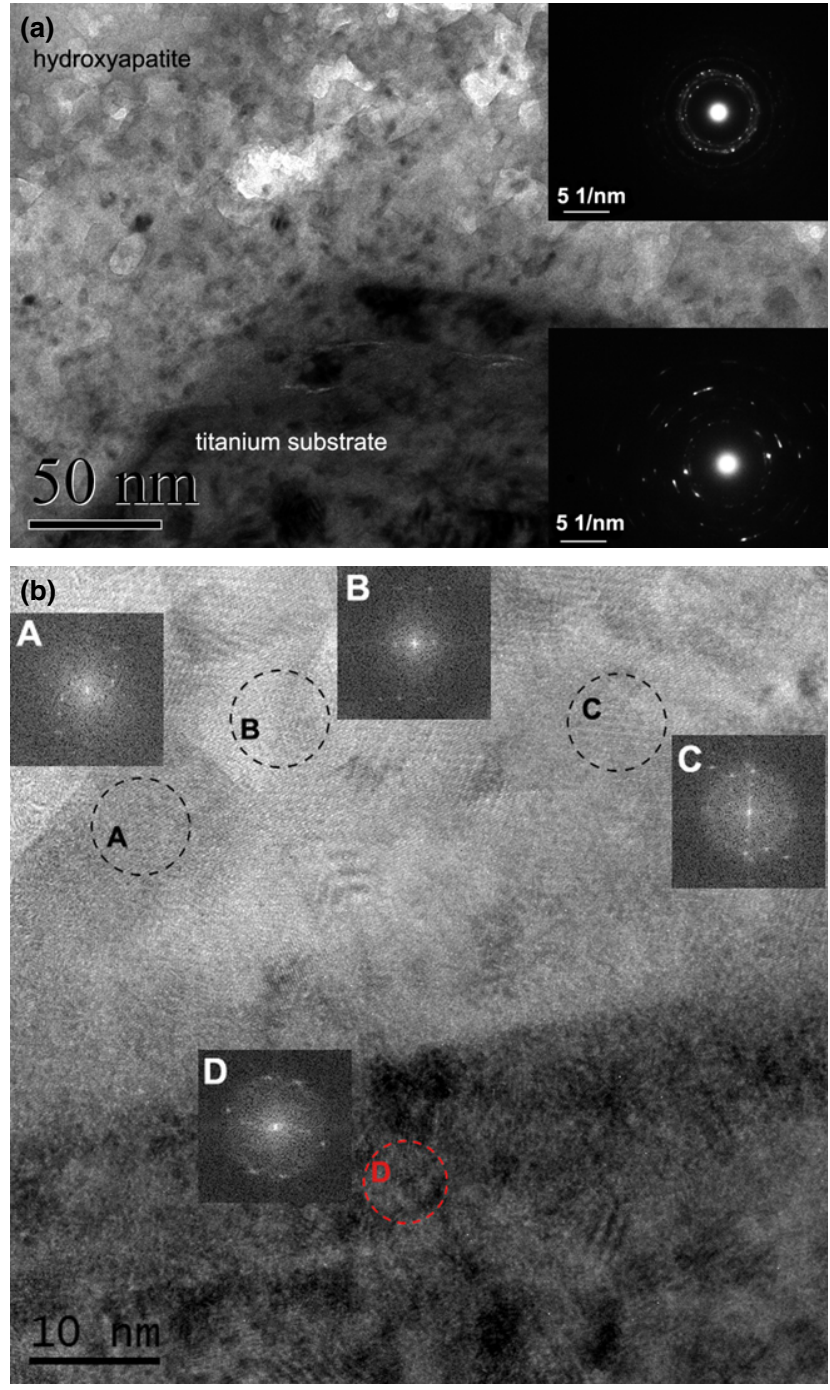

Fig. 7. (a) Hydroxyapatite-titanium alloy interface showing the fine-grain size at both sides of the interface, as well as the corresponding SAED patterns, (b) HRTEM of the interface with inserted FFT images.

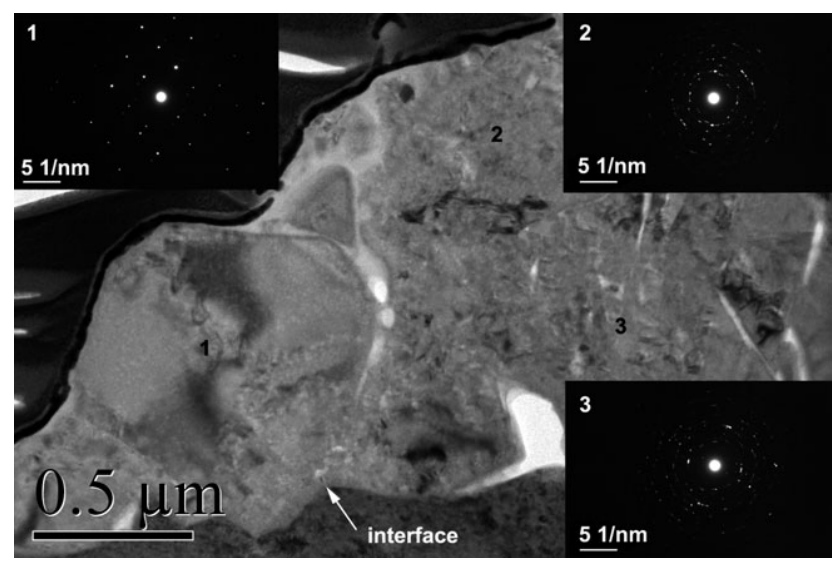

Fig. 8. TEM micrograph of a $7 \mu \mathrm{m}$ HA splat onto Ti6Al4V (inset SAED patterns).

structure is visible with predominance of very fine-grain regions, without the presence of plastic deformation features.

The overall impact features on a softer substrate were observed to be quite different. By performing similar analysis on the 7075-T6 aluminum alloy, no splats with a diameter less than $15 \mu \mathrm{m}$ could be found and the TEM evaluation of

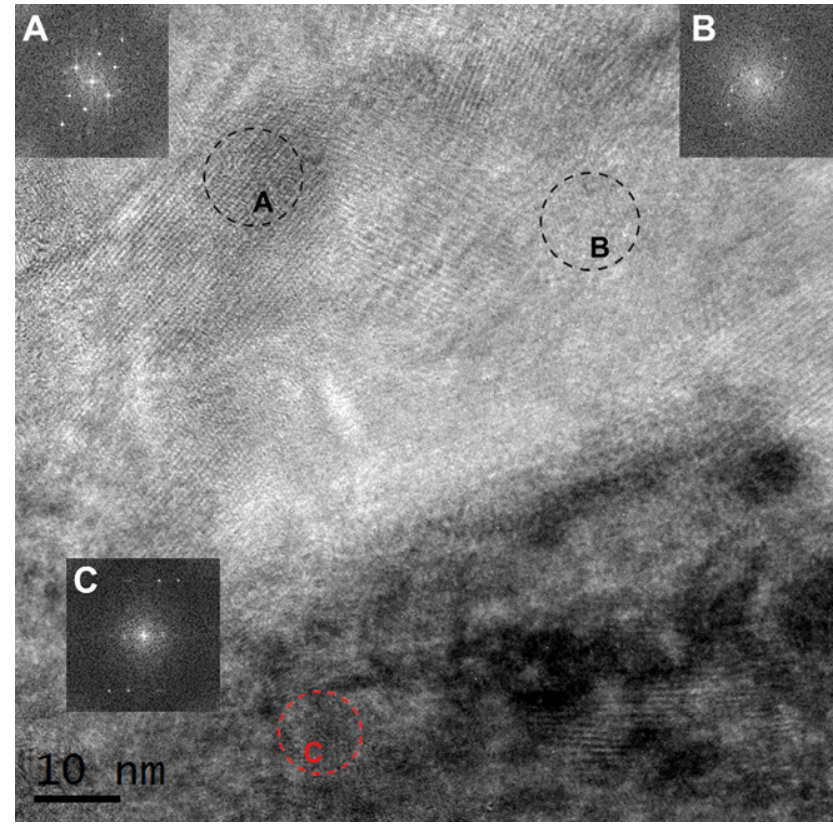

Fig. 9. HRTEM of the Hydroxyapatite-aluminum alloy interface with inserted FFT images.

a $17 \mu \mathrm{m}$ splat indicates extensive void collapse plus cracking and crushing. The aspect considerably changed from that typical from the original particles. Very small fragments were produced which appear densely packed. A large amount of small straight transgranular microcracks nucleated within the crushed zones, apparently much more than those corresponding to the $12 \mu \mathrm{m}$ splat on the titanium alloy. Some misorientation of about $10^{\circ}$ between pairs of submicrometer grains would demonstrate the presence of low-angle grain boundaries. The particle-substrate interface also shows grain refinement; the HRTEM image (Fig. 9) shows some Moirés patterns at both sides of the interface. Also, several FFT images from the HA (A, B, C) and the substrate (D) are presented. The identification of "D" also reveals the presence of spots corresponding to alumina, i.e., $\sim 0.245$ and $\sim 0.218 \mathrm{~nm}$.

Further observation of coarser splats on aluminum alloy allowed revealing the initial features of shock wave propagation with associated plastic deformation features. Figure 10 (low magnification) shows extinction contour at boundaries as well as a strong rotation at local regions. The final magnification in Fig. 10 indicates the presence within a grain of a dislocation network.

A more detailed work reporting the characteristics of a full coated surface is under performance; we can advance, however, that as result from all the detailed features described above, the resulting deposit exhibits a very different structure. The X-ray diffraction of the resulting cold-sprayed coating shows very broad peaks which are indicative of the reduced grain size upon particles impact (Fig. 11). In order to confirm the TEM results, a rietveld refinement was also here performed, leading to an average of $22.6 \mathrm{~nm}$ of crystallite size for the HA. This confirms that TEM and X-ray analysis lead to results of the same magnitude order.

\section{Discussion}

Although the deposition of ceramics has not been much investigated by CGS, their solid bonding phenomena has also been reported in other deposition techniques at room temperature such as aerosol deposition (AD), sometimes known as vacuum cold gas spray (VCGS) and nanoparticle deposition systems (NPDS), ${ }^{17-19,28,29}$ HA coatings have also been actually produced by this technology. ${ }^{30,31}$ In $\mathrm{AD}$, the impinged particles fracture on the substrate in analogy to the 


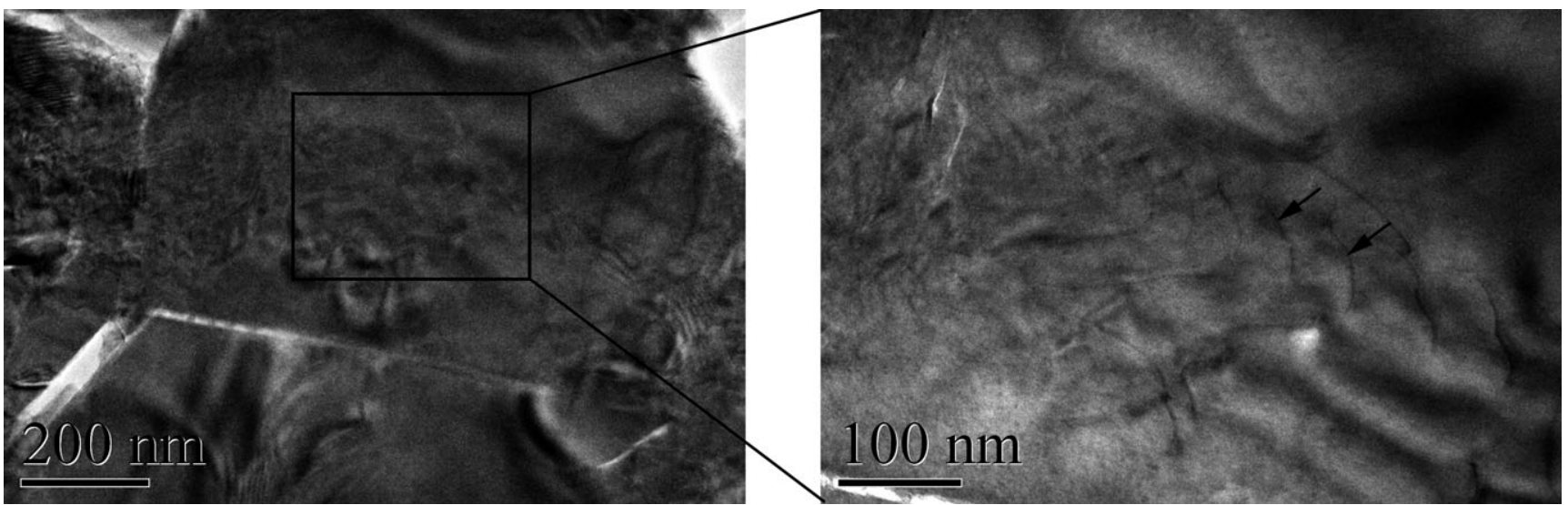

Fig. 10. Dislocation networks within a grain indicated by arrows at the magnified micrograph.

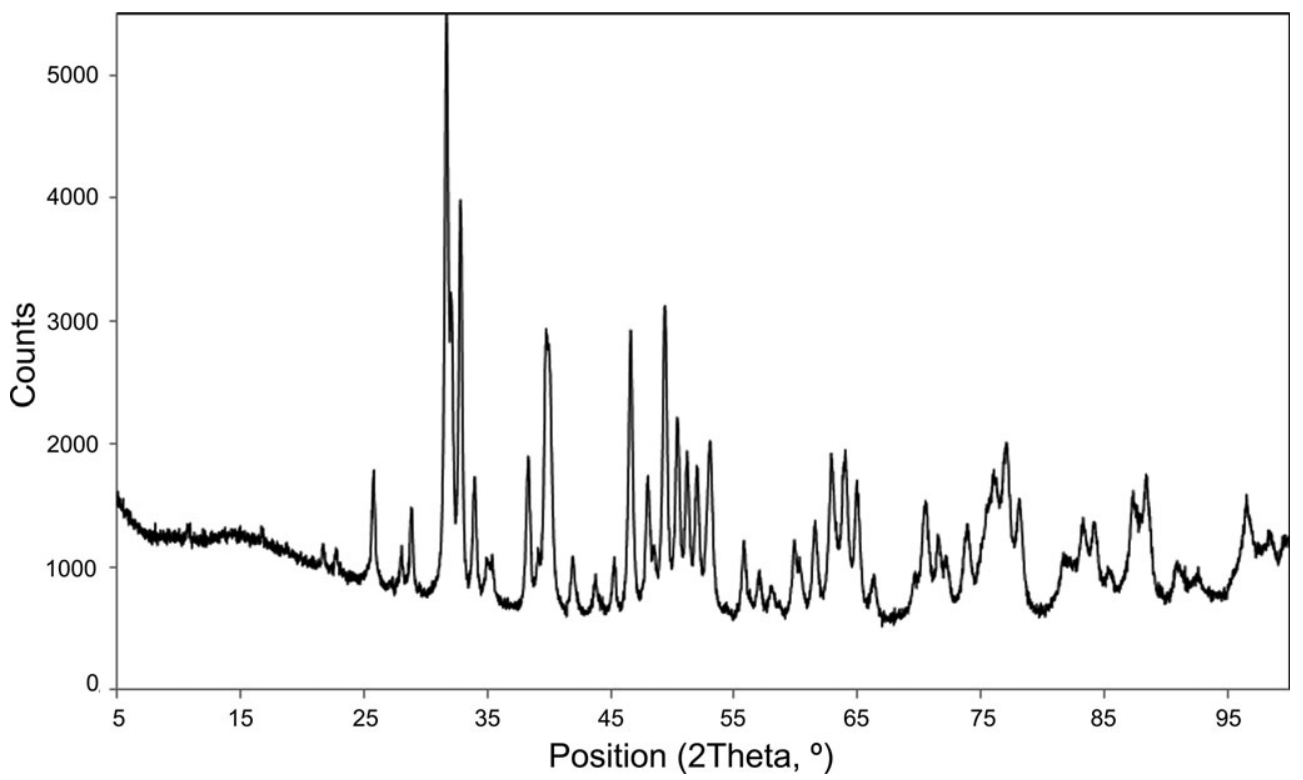

Fig. 11. X-ray diffraction of a hydroxyapatite cold-sprayed coating obtained from porous granular feedstock powder.

plastic deformation of a metallic particle. Then, the newly formed surface bonds to the substrate surface. This phenomenon is called room-temperature impact consolidation (RTIC) ${ }^{17}$ [Fig. 12(a)]. It resembles the dynamic fragmentation simulated by Park et al., ${ }^{32}$ where an alumina particle undergoes shock-induced plasticity and is finally fragmented in order to release the energy that the material cannot accommodate. These authors indicate that the film growth process is a competition between particle plasticity and fracture+erosion.

CGS is, however, different from AD and NPDS mainly in that the latter two, use submicrometer feedstock particles while usually particles $<50 \mu \mathrm{m}$ are used in CGS. A careful reading of the few articles published about CGS of ceramics indicates that the actual bonding mechanism is highly dependent on the microstructure of the feedstock powder and the relative particle-substrate hardness ratio, but the actual mechanism is not yet well clarified. This would explain why some authors observe the embedment of the ceramic particles into very soft and ductile substrates [Fig. 12(b)], whereas others, such as Yamada et al. ${ }^{16}$ support a chemical bonding [Fig. 12(c)] rather than anchoring. It appears that for dense powders, the effective deposition is either possible on soft substrates, where the embedment is possible but the posterior coating built-up is hardly likely ${ }^{12,13}$ or, on harder substrates through particle fragmentation. ${ }^{32}$ On soft substrates, only if substrate material is brought to the surface and is available to bind other particles, a second layer or parts of it are likely to be attached to the coating upon impact. On harder substrates, it is thought that the optimal particle state for a successful substrate attachment is that in which the Hugoniot Elastic Limit (HEL) of the ceramic is reached. ${ }^{32}$ The HEL of high-performance ceramics under plane shock wave propagation has generally been attributed to the generation and propagation of microcracks, plastic deformation, and possible phase transformations. ${ }^{25}$ If, as result of the spraying parameters, the shock loading pressure exceeds the HEL value, the plastic-fracture ratio decreases. Due to this increased fracture, the elastic rebound forces make that only small remnants of the brittle particle remain in the bonding zones. Most authors agree that the HEL for a ceramic provides a threshold below which the material retains some cohesion and has finite spall strength and above which the material has zero spall strength and becomes a confined powder, except for the work presented by Longy \& Cagnoux (1990), who reported that cohesion can be maintained above HEL. ${ }^{33}$

For porous or granular powders, the mechanisms may change since yielding threshold is known to decrease with decreasing density. Notice that Klieman et al. ${ }^{13}$ as well as Yamada et al. ${ }^{16}$ sprayed $\mathrm{TiO}_{2}$ onto stainless steel, achieving the second ones much thicker deposits; although the spraying tested conditions are slightly different for both studies, it is obvious that the intrinsic porosity of the powder used by the 
(a)

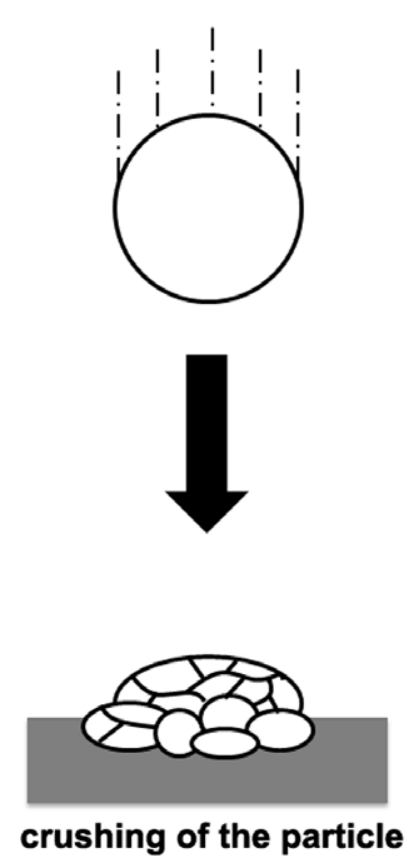

(b)

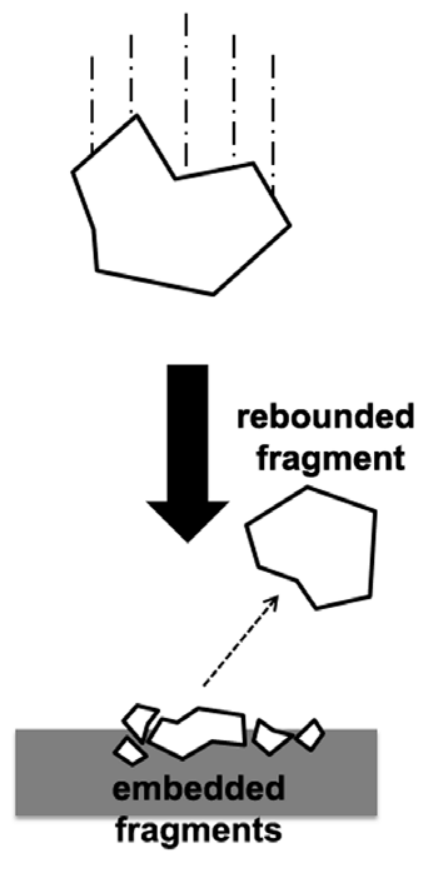

(c)

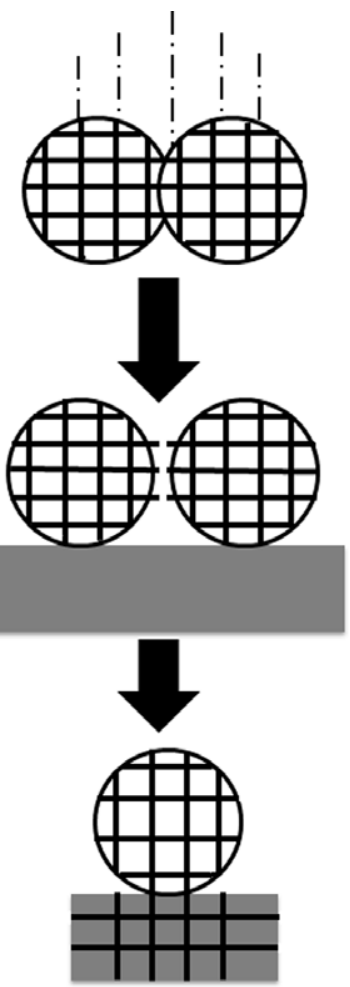

Fig. 12. Suggested bonding mechanisms of ceramic particles in low-temperatures deposition processes: (a) room-temperature impact consolidation (RTIC), (b) embedment or mechanical anchoring, (c) chemical bonding.

second group of authors is much more favorable for crack nucleation and, therefore, fragmentation and porous collapse. In addition, the nanoscaled primary particles configuring the porous powder seem to be also important to explain the chemical bonding mechanism.

The micrographs presented in the present work also seem to reveal the importance of the primary porous structure. In any of the substrates, their attachment leads to slight scattering of fragments around the central part, similar to the jets of metal splats, which has been commonly associated to adiabatic shearing instabilities when the material flows. However, it is obvious that the term "plastic flow" in ceramics cannot be understood as in metals due to the constraining conditions imposed by the surrounding material.

The crushing and pore collapsing observed in Fig. 6(d) has some similarities to the well-reported macroscopic examples of shock loading of rocks, ${ }^{34}$ this may be explained by the shock loading under high-strain rates occurring in microsized cold gas sprayed ceramic particles; this leads to strain localization and the typically shear band formation in inelastic solids. As assumed from the previous sections, strain localization in granular materials may not be the same as in fully dense materials. In granular materials, it can also take place normal to the principal compressive stress, forming socalled compaction or dilation bands. ${ }^{35}$ Curran et al. ${ }^{36}$ developed a micromechanical model for a granular material under high-strain rate deformation, with competition between dilatation and pore compaction.

At the microscale level, the propagation of the shock wave may proceed through microfracturing due to tensile and compressive stresses, as well as local shearing and twisting of grains, due to hydrostatic stress component, ${ }^{37}$ which would explain the contrast at the strained areas. The mechanism of fracturing might be well expected considering that, under the shock pressures given in CGS, the stress state in particles upon impact is much above the HEL limit of the material. ${ }^{38}$ Here, the presence of pores makes that the deformation occurs most likely first by fracture of the sintered necks between the grains. Grain boundaries of initial HA particles are the weak sites that easily induce intergranular microcrack formation, behaving as damage initiation points. Damage can further proceed in transgranular mode by shear and/or twist fracture at single or multiple planes in favorable oriented grains. $^{36}$

With the increased strain rates up to the levels occurring in CGS, slip is more difficult, and associated crack initiation may be suppressed, with a commensurate rise in strength, as observed from the force versus displacement curves at the flat punch tests. However, some cells and networks of dislocations were observed, which could be responsible for the work hardening of the material during dynamic deformation and become a competitive mechanism.

Although no temperature measurements on the particles can be undergone, depending on the local temperature rise upon impact, also recrystallization mechanisms might be involved, which would explain the grain refinement observed in Fig. 8 for the $7 \mu \mathrm{m}$ splat on Ti6Al4V. In this case, if this is the actual mechanism or it is purely given by shock-induced plastic deformation through strong rotation of a local region at boundaries through the formation of low-angle grain boundaries, would involve precise control of the kinetic energy and particle temperature. The differences observed for the 7 and $12 \mu \mathrm{m}$ splats appear to indicate how important is the particle size effect, as already predicted by the simulation work of Singh et al. ${ }^{21}$ The fact that the coarser HA splat on titanium alloy possessed still some porosity and that the extent of fine-grain areas is low means that it required higher shear stress for deformation mechanisms. In both cases, however, small and large splats, the coexistence of single-crystalline areas and polycrystalline areas rather than uniform reduction in crystallite size as in $\mathrm{AD}$, might be indicative of a nonuniform stress distribution upon impact when increasing particle size, with predominant grain refinement in smaller particles, as well as the pore distribution with respect to the direction of load application.

The decrease in particle size increases particle velocity and, therefore, higher extend of heat release upon impact which, 
joined with the low titanium thermal diffusivities may lead to the formation of new nuclei as a result of strong localized heating. Considering that gas temperature was below $400^{\circ} \mathrm{C}$, the local temperature rise upon impact should be high to induce ceramic recrystallization. ${ }^{38}$ This is also worth noting at the interface, which shows grain refinement also for the $12 \mu \mathrm{m}$ splat.

One striking feature is that, comparing analogous size splats, on the softer aluminum alloy substrate, the overall fragmentation process is much more extensive. The lower hardness of the substrate would imply a lower effective strain and thus, a lower flattening ratio for the same granular material since grain-boundary energies are the same. The results seem to indicate that the initial particle size was also determining the shock loading conditions. The larger presence of plastic deformation features on the coarser splat on aluminum indicates that they are not completely suppressed and become more competitive than in the case of the smaller splat.

The study of the interfaces by HRTEM indicates that grain refinement occurs as for the titanium substrate since these are the regions suffering from the higher shear stresses; however, native titanium and aluminum oxides appear not to remove at all by the impact of the porous HA particle. Whether they would do by the impact of more compact particles, as it occurs when cold spraying metallic powders, should be investigated.

Studies on AD and NPDS bonding mechanisms steeply proceeds, but further study needs to be done in CGS of ceramics and, given that those other two processes have achieved rather thick coatings, it is plausible to think that one of the most critical variables is particle size; ${ }^{21}$ smaller particle sizes would lead to easier densification.

Finally, the overall grain size reduction in HA may be favorable when immersed in body fluids due to the increased surface area, therefore facilitating ion release and posterior deposition of biomimetic apatites.

\section{Conclusions}

To sum up, at low temperature and high-strain rate processes such as cold gas spray, porous ceramics suffer from porous collapse of the structure and almost a complete lack of plastic deformation, due to the absence of mobile dislocation activity, although other modes of inelastic deformation, such as microcracking can provide alternative deformation mechanisms.

A larger extent of densification with considerable decrease in crystallite size has been observed at small splats, occurring where local rising temperature is much higher; therefore, dynamic crystallization might occur through local shearing and twisting of grains, as observed by the presence of some low-angle grain boundaries. It has been observed that the predominant mechanism, rather than the type of substrate, is mainly determined by the particle size, which is controlling the initial kinetic energy and, therefore, the shock loading conditions.

At particle-substrate interfaces, a dense stacking structure was found with fine HA crystallites whose presence may confirm the effect of localized heating upon impact.

\section{Acknowledgments}

The authors thank the Generalitat de Catalunya for the project 2014 SGR 1558

\section{References}

${ }^{1}$ L. Sun, C. C. Berndt, K. A. Gross, and A. Kucuk, "Material Fundamentals and Clinical Performance of Plasma-Sprayed Hydroxyapatite Coatings: A Review," J. Biomed. Mater. Res., 58, 570-92 (2001).

${ }^{2}$ A. W. Noorakma, H. Zuhailawati, V. Aishvarya, and B. K. Dhindaw, "Hydroxyapatite-Coated Magnesium-Based Biodegradable Alloy: Cold Spray
Deposition and Simulated Body Fluid Studies," J. Mater. Eng. Perform., 22 2997-3004 (2013)

${ }^{3}$ J. H. Lee, H. L. Jang, K. M. Lee, H.-R. Baek, K. Jin, and K. S. Hong, "In Vitro and in Vivo Evaluation of the Bioactivity of Hydroxyapatite-Coated Polyetheretherketone Biocomposites Created by Cold Spray Technology," Acta. Biomater., 9, 6177-87 (2013).

${ }^{4}$ V. K. Champagne, The Cold Spray Materials Deposition Process Fundamentals and Applications. Woodhead Publishing, Cambridge, UK, 2007 ISBN: 978-1-84569-181-3.

${ }^{5}$ H. Herman, S. Sampath, and R. McCune, "Thermal Spray: Current Status and Future Trends," MRS Bull., 25, 17-25 (2000).

${ }^{6}$ R. Ghelichi and M. Guagliano, "Coating by the Cold Spray Process: A State of the art," Frattura ed. Integrità Strutturale, 8, 30-44 (2009)

${ }^{7}$ S. Dosta, M. Couto, and J. M. Guilemany, "Cold Spray Deposition of a WC-25Co Cermet Onto Al7075-T6 and Carbon Steel Substrates," Acta. Mater., 61, 643-52 (2013).

${ }^{8}$ M. Yandouzi, E. Sansoucy, L. Ajdelsztajn, and B. Jodoin, "WC-Based Cermet Coatings Produced by Cold gas Dynamic and Pulsed gas Dynamic Spraying Processes," Surf. Coat. Tech., 202, 382-90 (2007).

${ }^{9}$ X. Zhou and P. Mohanty, "Electrochemical Behavior of Cold Sprayed Hydroxyapatite/Titanium Composite in Hanks' Solution," Electrochim. Acta., 65, $134-40$ (2012)

${ }^{10}$ V. Shukla, G. Elliott, B. Kear, and L. McCandlish, "Hyperkinetic Deposition of Nanopowders by Supersonic Rectangular jet Impingement," Scripta. Mater., 44, 2179-82 (2001).

${ }^{11} \mathrm{X}$. Zhou, "Hydroxyapatite/Titanium Composite Coating for Biomedical Applications", dissertation 2012.

${ }^{12}$ D. Seo, M. Sayar, and K. Ogawa, "SiO 2 and $\mathrm{MoSi}_{2}$ Formation on Inconel 625 Surface via SiC Coating Deposited by Cold Spray," Surf. Coat. Tech., 206, 2851-8 (2012).

${ }^{13}$ J.-O. Kliemann, H. Gutzmann, F. Gärtner, H. Hübner, C. Borchers, and T. Klassen, "Formation of Cold-Sprayed Ceramic Titanium Dioxide Layers on Metal Surfaces," J. Therm. Spray Tech., 20 [1-2] 292-8 (2011).

${ }^{14} \mathrm{~J}$. Vlcek, L. Gimeno, H. Huber, and E. Lugscheider, "A Systematic Approach to Material Eligibility for the Cold-Spray Process," J. Therm. Spray Tech., 14 [1] 125-33 (2005).

${ }^{15}$ C.-J. Li, G.-J. Yang, X.-C. Huang, W.-Y. Li, and A. Ohmori, "Formation of $\mathrm{TiO}_{2}$ Photocatalyst Through Cold Spraying"; pp. 315-19 In Thermal Spray 2004: Advances in Technology and Application, ASM International, Novelty, $\mathrm{OH}, 2004$.

${ }^{16} \mathrm{M}$. Yamada, H. Isago, K. Shima, H. Nakano, and M. Fukumoto "Deposition of $\mathrm{TiO}_{2}$ Ceramic Particles on Cold Spray Process"; pp. 172-6 in Thermal Spray 2010: Global Solutions for Future Applications, Springer-Verlag, New York, NY, 2011. ISBN 978-1-4419-8359-6.

${ }^{17}$ D.-M. Chun and S.-H. Ahn, "Deposition Mechanism of dry Sprayed Ceramic Particles at Room Temperature Using a Nano-Particle Deposition System", Acta. Mater., 59, 2693-703 (2011).

${ }^{18}$ D. Chun, J. Choi, C. S. Lee, and S. Ahn, "Effect of Stand-off Distance for Cold gas Spraying of Fine Ceramic Particles Under low Vacuum and Room Temperature Using Nano-Particle Deposition System (NPDS)," Surf. Coat. Tech., 206, 2125-32 (2012).

${ }^{19}$ J. Akedo, "Aerosol Deposition of Ceramic Thick Films at Room Temperture: Densification Mechanism of Ceramic Layers," J. Am. Ceram. Soc., 89, 1834-9 (2006)

${ }^{20}$ L. Zhang and W. T. Zhang, "Numerical Investigation on Particle Velocity in Cold Spraying of Hydroxyapatite Coating," Adv. Mater. Res., 18, 717-22 (2011).

${ }^{21}$ R. P. Singh and B. Batra, "Effect of Cold Spraying Parameters and Their Interaction an Hydroxyapatite Deposition," J. Appl. Fluid Mech., 6, 555-61 (2013).

${ }^{22}$ M. Grujicic, C. L. Zhao, W. S. DeRoseet, and D. Helfritch, "Adiabatic Shear Instability Based Mechanism for Particles/Substrate Bonding in the Cold-gas Dynamic-Spray Process," Mater. Design., 25, 681-8 (2004).

${ }^{23}$ Y. V. R. K. Prasad, "Processing Maps: A Status Report," J. Mater. Eng. Perform., 12, 638-45 (2003).

${ }^{24}$ C. Gandhi and M. F. Ashby, "Fracture-Mechanism Maps for Materials Which Cleave: FCC, BCC and HCP Metals and Ceramics," Acta. Metal., 27, 1565-602 (1979)

${ }^{25}$ M. W. Chen, J. W. McCauley, D. P. Dandekar, and N. K. Bourne, "Dynamic Plasticity and Failure of High-Purity Alumina Under Shock Loading," Nat. Mater., 5, 614-18 (2006).

${ }^{26}$ B. Dodd and Y. Bai, Adiabatic Shear Localization, 2nd ed. Elsevier, Waltham, 2012. ISBN-9780080982007.

${ }^{27}$ A. M. Vilardell, N. Cinca, S. Dosta, I. G. Cano, and J. M. Guilemany, "Multifunctional Miro-Nano Structured Hydroxyapatit Coatings Onttianum Ti6Al4V for Biomedical Applications by Cold Gas Spray," Acta. de Protocolarización num., 809 (2015).

${ }^{28} \mathrm{~J}$. Akedo, "Room Temperature Impact Consolidation (RTIC) of Fine Ceramic Powder by Aerosol Deposition Method and Applications to Microdevices," J. Therm. Spray Tech., 17 [2] 181-98 (2008).

${ }^{29} \mathrm{~T}$. Miyoshi, "Preparation of Full-Dense $\mathrm{Pb}(\mathrm{Zr}, \mathrm{Ti}) \mathrm{O} 3$ Ceramics by Aerosol Deposition," J. Am. Ceram. Soc., DVS-26491 [7] 2098-104 (2008).

${ }^{30}$ B. Hahn, et al. , "Dense Nanostructured Hydroxyapatite Coating on Titanium by Aerosol Deposition," J. Am. Ceram. Soc., 92 [3] 683-7 (2009).

${ }^{31}$ D. Park, et al. , "Improved Biocompatibility of Hydroxyapatite Thin Film Prepared by Aerosol Deposition," J. Biomed. Mater. Res. B: Appl. Biomat., 94B [2] 353-8 (2010).

${ }^{32}$ H. Park, F. Cao, J. Kwon, and C. Lee, "The Effect of Schock-Induced Plastic Deformation on Alumina Deposition During Vacuum Kinetic Spraying"; pp. 279-85 in Thermal Spray 2013: Innovative Coating Solutions for the 
Global Economy, ASM International, Novelty, OH, 2014. ISBN 978-1-63266681-9.

${ }^{33}$ F. Longy and J. Cagnoux, "Plate-Impact Recovery Experiments of Ceramics"; pp. 441-4 in APS Proc-Shock Waves in Condensed Matter, Edited by S. C. Schmidt, J. N. Johnson, and L. W. Davison. Elsevier, Waltham, 1989.

${ }^{34}$ A. El Bied, J. Sulem, and F. Martineau, "Microstructure of Shear Zones in Fontainebleau Sandstone," Int. J. Rock Mech. Min. Sci., 39 [7] 917-32 (2002).

${ }^{35}$ X. Du Bernard, P. Eichhubl, and A. Aydin, "Dilation Bands: A new Form of Localized Failure in Granular Media," Geophys. Res. Lett., 29 [24] 4pp (2002).
${ }^{36}$ D. R. Curran, L. Seaman, T. Copper, and D. A. Shockey, "Micromechanical Model for Comminution and Granular Flow of Brittle Material Under High Strain Rate Application to Penetration of Ceramic Targets," Int. J. Impact Eng., 13, 53-83 (1993).

${ }^{37}$ L. He, O. C. Standard, T. T. Y. Huang, B. A. Latella, and M. V. Swain, "Mechanical Behavior of Porous Hydroxyapatite," Acta. Biomater., 4, 577-86 (2008).

${ }^{38}$ N. V. Ivchenko, R. I. Kuznetsov, and B. B. Beresnev, "Effect of Hydrostatic Pressure on the Recrystallization of CsJ(T1) Crystals," Fiz. Tekh. Vysokikh Davlenii, 21, 30-7 (1986). 